\title{
Adjunctive markers for classification and diagnosis of central nervous system tumors: results of a multi-center neuropathological survey in Korea
}

\author{
Yoon Jin Cha ${ }^{1 *}$, Se Hoon $\mathrm{Kim}^{1 *}$, Na Rae Kim², The Neuropathology Study Group of the Korean Society of Pathologists \\ 'Department of Pathology, Yonsei University College of Medicine, Seoul; \\ ²Department of Pathology, Gachon University Gil Medical Center, Incheon, Korea
}

\begin{abstract}
Background: The revised 4th 2016 World Health Organization (WHO) classification of tumors of the central nervous system (CNS) classification has adopted integrated diagnosis encompassing the histology and molecular features of CNS tumors. We aimed to investigate the immunohistochemistry, molecular testing, and testing methods for diagnosis of CNS tumors in pathological labs of tertiary centers in Korea, and evaluate the adequacy of tests for proper diagnosis in daily practice. Methods: A survey, composed of eight questions concerning molecular testing for diagnosis of CNS tumors, was sent to 10 neuropathologists working in tertiary centers in Korea. Results: For diagnosis of astrocytic and oligodendroglial tumors, all 10 centers performed isocitrate dehydrogenase mutations testing and $1 p / 19 q$ loss of heterozygosity. For glioneuronal tumors, immunohistochemistry (IHC) assays for synaptophysin $(n=9), C D 34(n=7)$, BRAF(VE1) $(n=5)$ were used. For embryonal tumors, particularly in medulloblastoma, four respondents used IHC panel (growth factor receptor bound protein 2-associated protein 1, filamin A, and yes-associated protein 1) for molecular subclassification. Regarding meningioma, all respondents performed $\mathrm{Ki}-67 \mathrm{IHC}$ and five performed telomerase reverse transcriptase promoter mutation. Conclusions: Most tertiary centers made proper diagnosis in line with 2016 WHO classification. As classification of CNS tumors has evolved to be more complex and more ancillary tests are required, these should be performed considering the effect of necessity and justification.
\end{abstract}

Key Words: World Health Organization; Central nervous system; Neoplasms; Molecular testing; Korea

Received: January 7, 2020 Revised: January 29, 2020 Accepted: February 4, 2020

Corresponding Author: Na Rae Kim, MD, Department of Pathology, Gachon University Gil Medical Center, 21 Namdong-daero 774beon-gil, Namdong-gu, Incheon 21565, Korea Tel: +82-32-460-3073, Fax: +82-32-460-2394, E-mail: clara_nrk@gilhospital.com

*Yoon Jin Cha and Se Hoon Kim contributed equally to this work.

The 4th revised World Health Organization (WHO) classification of tumors of the central nervous system (CNS) highlights the importance of integrated diagnosis of CNS tumors [1]. In addition to histological features, molecular signatures are now mandatory for diagnosis, as well as patient management in neuro-oncological aspects. Molecularly different tumors could have different biological behavior and treatment response to therapeutic agents. The 2016 update of WHO classification suggests well-established molecular parameters in diagnostic algorithms of diffuse gliomas. For appropriate integrated diagnosis of diffuse gliomas, one needs to examine the isocitrate dehydrogenase (IDH) mutation and 1p/19q status. In this study, we investigated current adjuvant examination being performed in Korea for diagnosis of brain tumors.

\section{MATERIALS AND METHODS}

The survey was distributed to the members of the Korean Society of Neuropathology ( $\mathrm{n}=12$ ) from May to June 2017 (30 days). We expected that most institutions would have prepared for the new diagnostic criteria proposed by the revised WHO classification.

Survey questionnaires included the number of neuropathological specimens per year, ancillary tests being performed for brain tumor diagnosis, and the type of methods for the ancillary tests. The results were analyzed after receiving answers to the survey.

\section{Ethics statement}

This study did not involve human subjects and was approved 
by the Institutional Review Board of Gachon University Gil Medical Center with a waiver of informed consent (GFIRB2018-266).

\section{RESULTS}

The survey questions were answered by 10 of the 12 neuropathologists who received the survey questionnaires. All 10 respondents were neuropathologists working in tertiary centers capable of neurosurgery and neuropathological diagnosis. The total number of neuropathological cases for a year, including tumor, non-tumor, muscle, and peripheral nerve biopsy, was more than 300 cases in five centers (average, 906; range, 392 to 1,900), 200-300 cases in one center, 100-200 cases in another, 50-100 in two, and less than 50 cases in two others. All 10 centers make the diagnosis based on the 4th revised 2016 WHO classification.

\section{Immunohistochemical stainings in gliomas}

For diagnosis of astrocytic and oligodendroglial tumors, immunohistochemistry (IHC) for glial fibrillary acidic protein (GFAP), oligodendrocyte transcription factor 2 (OLIG2), $\alpha$-thalassemia/ mental retardation syndrome X-linked (ATRX), IDH1, BRAF (VE1), Ki-67, p53, S-100, vimentin, epidermal growth factor receptor (EGFR), synaptophysin, H3.3K27M, and H3.3K27me3 was used. Depending on the centers, three to 11 markers were used for diagnosis. The most commonly used IHC markers were IDH1, ATRX, p53, and Ki-67 (Fig. 1A).

In pilocytic astrocytomas and pleomorphic xanthoastocyto- mas (PXAs), most IHC markers were overlapped with the items mentioned above (Supplementary Fig. S1).

For diagnosis of ependymomas, GFAP, epithelial membrane
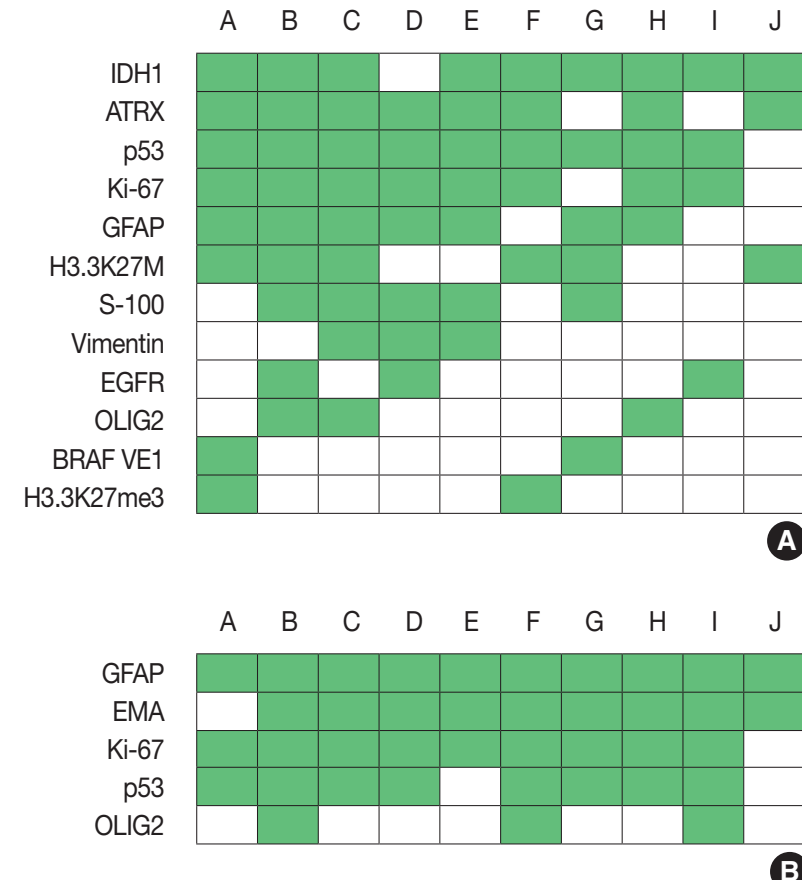

Fig. 1. Immunohistochemistry markers used in glioma (A) and ependymoma (B). Alphabet A-J represents respondents. IDH1, isocitrate dehydrogenase 1; ATRX, $\alpha$-thalassemia/mental retardation syndrome X-linked; GFAP, glial fibrillary acidic protein; EGFR, epidermal growth factor receptor; OLIG2, oligodendrocyte transcription factor 2; EMA, epithelial membrane antigen.
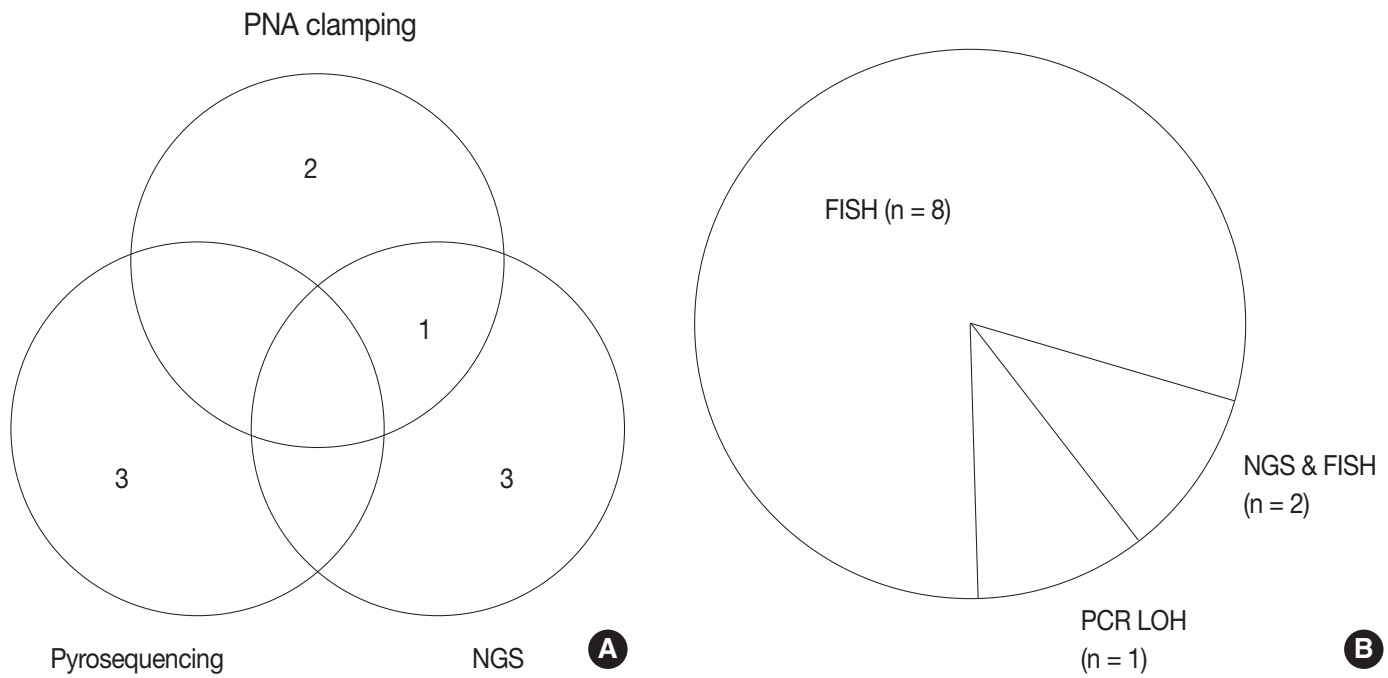

Fig. 2. Current methods for isocitrate dehydrogenase $(\mathrm{IDH})$ mutation test $(A)$ and $1 p / 19 q$ status $(B)$ among respondents. PNA, peptide nucleic acid; NGS, next-generation sequencing; FISH, fluorescence in situ hybridization; PCR, polymerase chain reaction; LOH, loss of heterozygosity. 
antigen, $\mathrm{Ki}-67$, and $\mathrm{p} 53$ were most frequently used, followed by CD99 and OLIG2 (Fig. 1B).

\section{Molecular testing in gliomas}

Regarding astrocytic and oligodendroglial tumors, IDH mutation tests (including IDH1 and IDH2) (n=9) and 1p/19q loss of heterozygosity $(\mathrm{LOH})(\mathrm{n}=10)$ were performed before making a final diagnosis. Next-generation sequencing (NGS) $(n=4)$, pyrosequencing $(\mathrm{n}=3)$, peptide nucleic acid (PNA) clamping method $(\mathrm{n}=3)$, and Sanger sequencing $(\mathrm{n}=1)$ methods were used for IDH mutation testing (Fig. 2A). One center performs both NGS and PNA clamping methods for IDH mutation test whereas the other one uses only IDH1 IHC. While most of the centers used fluorescence in situ hybridization (FISH) to evaluate the $1 \mathrm{p} / 19 \mathrm{q}$ status, two centers conducted NGS in addition to FISH, for evaluating the $1 \mathrm{p} / 19 \mathrm{q}$ status. One center used polymerase chain reaction-based $\mathrm{LOH}$ analysis (Fig. 2B)

Additional molecular testing included MGMT ( $\mathrm{O}^{6}$-methylguanine-DNA methyltransferase) methylation test $(\mathrm{n}=8)$, and telomerase reverse transcriptase (TERT) promoter mutation $(\mathrm{n}=$ 8; four single test, four NGS). BRAF mutation test and NGS test, for V600E, were performed in eight centers. One center separately performed $B R A F$ mutation test in all astrocytic and oligodendroglial tumors. Two centers performed BRAF mutation test in all infiltrating gliomas except oligodendroglioma.

For pilocytic astrocytomas and PXAs, most molecular testings were overlapped with the items mentioned above (Supplementary Fig. S2).

For ependymomas, three centers performed NGS and one center performed an additional FISH test for 1q gain.

\section{Ganglioglioma and dysembryoplastic neuroepithelial tumor}

Regarding glioneuronal tumors, the survey focused on ganglioglioma and dysembryoplastic neuroepithelial tumor (DNET), as they comprised the majority of glioneuronal tumors. In cases of ganglioglioma, the most commonly used IHC markers were GFAP, Ki-67, and synaptophysin, followed by NeuN and CD34 (Fig. 3A), whereas for DNET, synaptophysin, GFAP, Ki67, CD34, and NeuN were the most commonly used (Fig. 3B). Regarding molecular testing, one center performed the same molecular tests as in gliomas. In some centers, IDH mutation test ( $\mathrm{n}=3$ in ganglioglioma, $\mathrm{n}=2$ in DNET), BRAF V600E test ( $\mathrm{n}=1$ in ganglioglioma, $\mathrm{n}=2$ in DNET), and MGMT methylation test ( $\mathrm{n}=2$ in ganglioglioma, $\mathrm{n}=3$ in DNET) were performed. NGS was performed for ganglioglioma in three centers and DNET in one. Five centers did not perform further molec-
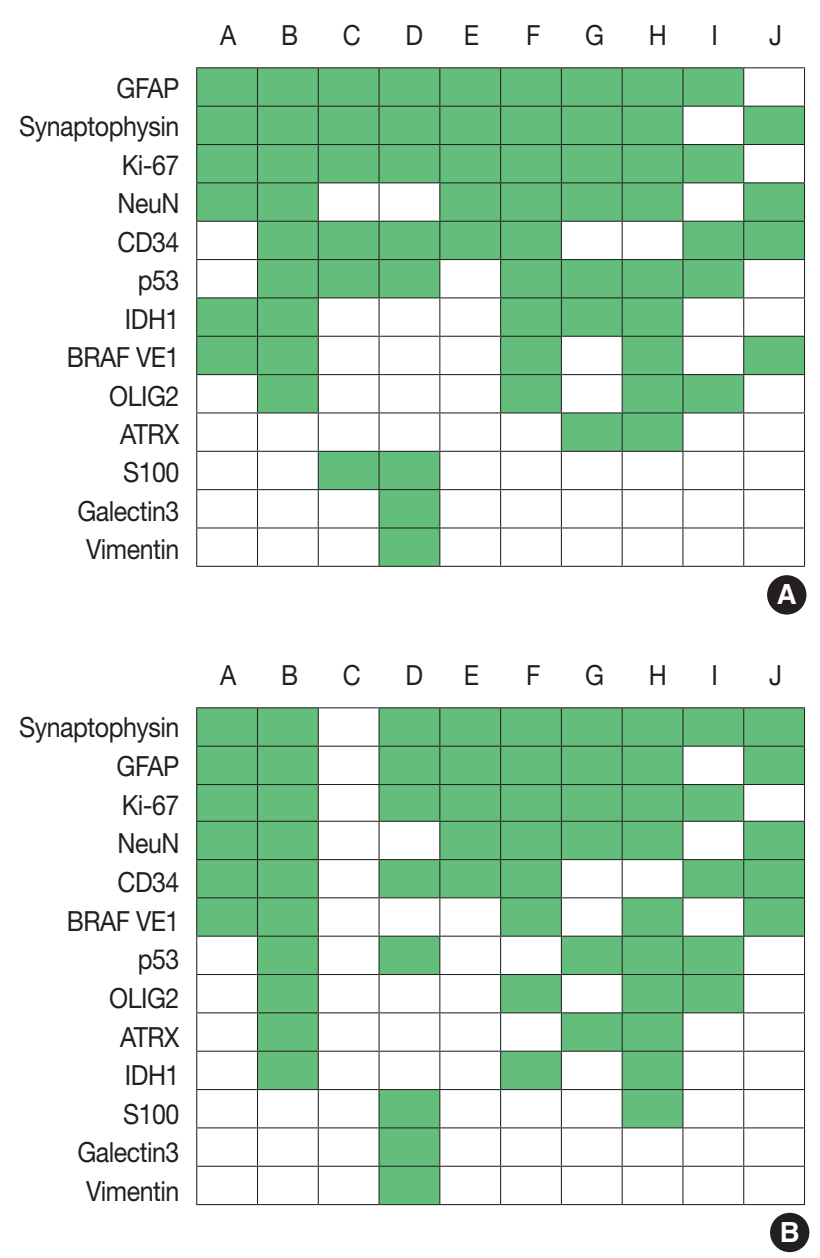

Fig. 3. Immunohistochemistry markers used in ganglioglioma (A) and dysembryoplastic neuroepithelial tumor (B). Alphabet A-J represents respondents. GFAP, glial fibrillary acidic protein; IDH1, isocitrate dehydrogenase 1; OLIG2, oligodendrocyte transcription factor 2; ATRX, $\alpha$-thalassemia/mental retardation syndrome Xlinked.

ular testing in ganglioglioma and DNET.

\section{Embryonal tumors}

p53 ( $\mathrm{n}=9), \mathrm{Ki}-67(\mathrm{n}=9)$, and synaptophysin $(\mathrm{n}=8)$ were the most commonly used IHC markers in medulloblastoma, followed by GFAP. For molecular classification, surrogate IHC markers, including growth factor receptor bound protein 2-associated protein $1(\mathrm{GAB} 1 ; \mathrm{n}=5), \beta$-catenin $(\mathrm{n}=1)$, filamin $A$ ( $\mathrm{n}=$ $4)$, and yes-associated protein 1 (YAP1) $(n=4)$, were used. All 10 centers performed integrase interactor 1 IHC for atypical teratoid rhabdoid tumors and one center performed additional BRG2 IHC. LIN28A IHC was used for embryonal tumors in three centers. Three centers performed NGS for embryonal tumors. 


\section{Meningioma}

All 10 centers performed Ki-67 IHC for meningioma, and additional phosphohistone $\mathrm{H} 3 \mathrm{IHC}$ was used in six centers. Three centers separately examined the TERT promoter mutation. Two centers performed NGS for meningioma, which included TERT in NGS panel.

\section{DISCUSSION}

We conducted surveys in 10 pathology labs in tertiary centers in Korea to investigate the current status of adjunctive examination for CNS tumor diagnosis. The 4th revised 2016 WHO classification adopted layered diagnosis encompassing histological and molecular features, which can precisely classify CNS tumors, thus allowing for more accurate predictions, regarding treatment response and prognosis [1]. In particular, IDH mutation and $1 \mathrm{p} / 19 \mathrm{q}$ status are the most important diagnostic discriminators of astrocytic and oligodendroglial tumors.

We found that the majority of tertiary centers examined IDH mutation and $1 \mathrm{p} / 19 \mathrm{q}$ status before development of final diagnosis in glial tumors. Particularly, IDH1 IHC is included in routine IHC panel in most centers and appears to be a very helpful surrogate marker for IDH mutation, because approximately over $90 \%$ of IDH mutation is IDH1 [2]. Although ATRX is not essential in diagnosis of glial tumors based on the current WHO classification, $80 \%$ of respondents routinely used ATRX IHC.

The ATRX gene is a telomere maintenance-related gene that functions in chromatic remodeling and maintains genomic stability incorporating $\mathrm{H} 3.3$ histone proteins into telomeres [3,4]. ATRX gene inactivation is correlated with the alternative lengthening of telomeres. A subset of gliomas harboring ATRX mutation is characterized by IDH mutation and $1 \mathrm{p} / 19 \mathrm{q}$ intact [57]. Loss of nuclear expression by IHC is a highly sensitive and specific feature of ATRX alteration [4] and can be used as a surrogate marker for ATRX mutation. Combining IHC panel with IDH1 and ATRX helps to predict astrocytic tumors in cases of those lacking ATRX expression [8].

MGMT methylation and TERT promoter mutation are not required for diagnosis, but impact patient prognosis and treatment response [9-11]. Promoter methylation of MGMT induces epigenetic silencing of the MGMT gene, restoring alkylated DNA. Temozolomide is an alkylating agent used in glioblastoma and the benefits of temozolomide treatment have been shown in patients with MGMT methylation [10]. TERT promoter mutation increases telomerase activity and is frequently found in glioblastomas and oligodendrogliomas [12]. Previous study classified gliomas into molecular groups based on IDH mutation, $1 \mathrm{p} / 19 \mathrm{q}$, and TERT mutation status [9]. Results showed higher survival rate in TERT and IDH mutation groups in grade II and III gliomas, whilst single TERT mutation showed the worst survival rate of all gliomas [9]. MGMT methylation and TERT promoter mutation tests were conducted in $80 \%$ of the respondents, and four centers obtained TERT promoter mutation results by NGS.

NGS has been evolving rapidly and is being adopted in many tertiary centers in Korea. NGS uses targeted gene panels to analyze hundreds of genes. In Korea, there are essential genes such as HER2, EGFR, ALK, KRAS, NRAS, BRAF, BRCA1, BRCA2, KIT, PDGFRA, IDH1, IDH2, MYC (C-myc), and Nmyc (MYCN) for non-hereditary solid tumors, that should be included in said panels. Most tertiary centers running NGS use gene panels with 50-300 genes. For gliomas, only one of the respondents was using a separate neuropathology gene panel, and the remaining were using a common panel for solid tumors. For infiltrating gliomas, NGS was routinely performed in six centers. In addition to EGFR, BRAF, IDH1, and IDH2 which are essential panel genes, six respondents had TERT, TP53, and SMARCB1 also in their panels, and one institution substituted FISH with 1p/19q LOH (by evaluation of microsatellite loci) by NGS.

For the diagnosis of glioneuronal tumors, CD34 and synaptophysin IHC were the most commonly performed assays. Synaptophysin is a marker for presynaptic vesicle, and is widely used to detect neuron or neuronal differentiation. DNET and ganglioglioma are the most common glioneuronal tumors and the differential diagnosis between these two is important, because DNET is a benign tumor but ganglioglioma could undergo malignant transformation $[13,14]$. Expression of CD34 is the one characteristic feature that favors ganglioglioma diagnosis over DNET [13]. BRAF V600E mutation could be found variably in glioneuronal tumors including DNET, ganglioglioma, and PXA $[15,16]$ and could be used as a diagnostic and targetable marker.

For meningioma, all respondents performed Ki-67 IHC routinely. Although Ki-67 labeling index (LI) is not included in the current grading system, $\mathrm{Ki}-67 \mathrm{LI}$ is correlated with mitosis and tumor recurrence $[17,18]$. In previous studies, tumors with more than $3 \%$ proliferative index, showed a shorter recurrence time after resection [19]. TERT mutation also has been reported in meningiomas, especially in recurrent and high grade (grades II and III) tumors [20]. Although an overall incidence of TERT mutation is low (around 5\%) in meningioma, TERT mutation 
predicted more aggressive behavior and poor prognosis in meningioma [21,22].

Medulloblastoma is one of the most common embryonal tumors and the second most common CNS tumor in children. Medulloblastoma is considered a heterogeneous group that can be classified into four molecular subgroups based on Sonic hedgehog (SHH) and WNT status. In daily practice, using three (GAB1, filamin A, and YAP1) or four IHC (GAB1, filamin A, YAP1, and $\beta$-catenin) panel could discriminate SHH, WNT, and non-SHH/WNT subgroups [23]. Moreover, TP53 mutation can designate the WNT type, IHC of p53 in mutant pattern could help to limitedly predict the molecular subtype [24]. Five respondents (50\%) used GAB1 and four used a panel composed of GAB1, filamin A, and YAP1, and nine respondents used $\mathrm{Ki}-67$ and $\mathrm{p} 53$ as routine IHC panel in medulloblastoma.

NGS can evaluate clinically significant variants across hundreds of targeted genes. In Korea, CNS tumor is classified under non-genetic solid tumor category. Among the essentially required 14 genes, EGFR, BRAF, IDH1, and IDH2 are relevant to $\mathrm{CNS}$ tumors. In the present survey, almost all tertiary centers except one used commercial panels and platforms from the same manufacturers, which also meets the government's minimum requirements. NGS is an accurate and sensitive tool for detection of additional genetic alteration with much less amount of DNA or RNA compared to the conventional techniques, which require more amount of tissue and only detect one alteration in one test. However, in daily practice, NGS is still an expensive ancillary test as it has a $2-4$ week-turnaround time. For prompt diagnosis and subsequent proper treatment, IDH mutation and $1 \mathrm{p} / 19 \mathrm{q} \mathrm{LOH}$ are being examined separately to achieve more rapid final diagnosis.

In conclusion, we found that most tertiary centers handling neuropathological specimens were making diagnosis according to the 2016 WHO classification. However, a limitation is still present because present survey could not encompass every single pathology lab in Korea. Although most CNS tumors in Korea are expected to be managed in tertiary centers that participated in this survey, there is a possibility that some cases that are not handled in tertiary centers may not be properly examined for the basic molecular status such as IDH mutation. For the sake of proper diagnosis, the first line of ancillary tests composed of IHC and simple molecular tests should be established. The second line of ancillary tests should encompass NGS assay and other further tests that can cover the detailed analysis of CNS tumors.

\section{Supplementary Materials}

The Data Supplement is available with this article at https:// doi.org/10.4132/jptm.2020.02.04.

\section{ORCID}

Yoon Jin Cha: https://orcid.org/0000-0002-5967-4064

Se Hoon Kim: https://orcid.org/0000-0001-7516-7372

Na Rae Kim: https://orcid.org/0000-0003-2793-6856

\author{
Author Contributions \\ Conceptualization: NRK, SHK. \\ Data curation: YJC. \\ Formal analysis: YJC. \\ Funding acquisition: NRK. \\ Investigation: YJC, SHK, NRK. \\ Methodology: SHK. \\ Supervision: SHK, NRK. \\ Validation: YJC. \\ Writing—original draft: YJC, SHK. \\ Writing—review \& editing: YJC, SHK, NRK.
}

\section{Conflicts of Interest}

S.H.K., contributing editor of the Journal of Pathology and Translational Medicine, was not involved in the editorial evaluation or decision to publish this article. All remaining authors have declared no conflicts of interest.

\section{Funding}

This research was supported by 2015 The Korean Society of Pathologists Grant.

\section{Acknowledgments}

We have to express our appreciation to the participants who generously shared their time, experience, and materials for the purposes of this project. All the products of this work would not be possible without their support and participation: Sung-Hye Park, Seoul National University Hospital; Youn Soo Lee, Seoul ST. Mary's Hospital; Mi Jung Kwon, Hallym University Sacred Heart Hospital; Sang Pyo Kim, Keimyung University Dongsan Medical Center; Eun Deok Chang, Uijeongbu ST. Mary's Hospital; Kyung Hwa Lee, Chonnam National University Hwasun Hospital; So Dug Lim, Konkuk University Medical Center; Soo Jeong Nam, Asan Medical Center.

\section{REFERENCES}

1. Louis DN, Ohgaki H, Wiestler OD, Cavenee WK. WHO classifica- 
tion of tumours of the central nervous system. 4th rev. ed. Lyon: IARC Press, 2016.

2. Yan H, Parsons DW, Jin G, et al. IDH1 and IDH2 mutations in gliomas. N Engl J Med 2009; 360: 765-73.

3. Pekmezci M, Rice T, Molinaro AM, et al. Adult infiltrating gliomas with WHO 2016 integrated diagnosis: additional prognostic roles of ATRX and TERT. Acta Neuropathol 2017; 133: 1001-16.

4. Heaphy CM, de Wilde RF, Jiao Y, et al. Altered telomeres in tumors with ATRX and DAXX mutations. Science 2011; 333: 425.

5. Kannan K, Inagaki A, Silber J, et al. Whole-exome sequencing identifies ATRX mutation as a key molecular determinant in lowergrade glioma. Oncotarget 2012; 3: 1194-203.

6. Leeper HE, Caron AA, Decker PA, Jenkins RB, Lachance DH, Giannini C. IDH mutation, 1p19q codeletion and ATRX loss in WHO grade II gliomas. Oncotarget 2015; 6: 30295-305.

7. Liu XY, Gerges N, Korshunov A, et al. Frequent ATRX mutations and loss of expression in adult diffuse astrocytic tumors carrying IDH1/IDH2 and TP53 mutations. Acta Neuropathol 2012; 124: 61525 .

8. Karsy M, Guan J, Cohen AL, Jensen RL, Colman H. New molecular considerations for glioma: IDH, ATRX, BRAF, TERT, H3 K27M. Curr Neurol Neurosci Rep 2017; 17: 19.

9. Eckel-Passow JE, Lachance DH, Molinaro AM, et al. Glioma groups based on $1 p / 19 q, I D H$, and TERT promoter mutations in tumors. N Engl J Med 2015; 372: 2499-508.

10. Hegi ME, Diserens AC, Gorlia T, et al. MGMT gene silencing and benefit from temozolomide in glioblastoma. N Engl J Med 2005; 352: 997-1003.

11. Stupp R, Hegi ME, Mason WP, et al. Effects of radiotherapy with concomitant and adjuvant temozolomide versus radiotherapy alone on survival in glioblastoma in a randomised phase III study: 5-year analysis of the EORTC-NCIC trial. Lancet Oncol 2009; 10: 459-66.

12. Arita H, Narita Y, Fukushima S, et al. Upregulating mutations in the TERT promoter commonly occur in adult malignant gliomas and are strongly associated with total 1p19q loss. Acta Neuropathol 2013; 126: 267-76.

13. Blumcke I, Wiestler OD. Gangliogliomas: an intriguing tumor entity associated with focal epilepsies. J Neuropathol Exp Neurol 2002; 61: 575-84.

14. Luyken C, Blumcke I, Fimmers R, Urbach H, Wiestler OD, Sch- ramm J. Supratentorial gangliogliomas: histopathologic grading and tumor recurrence in 184 patients with a median follow-up of 8 years. Cancer 2004; 101: 146-55.

15. Chappe C, Padovani L, Scavarda D, et al. Dysembryoplastic neuroepithelial tumors share with pleomorphic xanthoastrocytomas and gangliogliomas $B R A F(V 600 \mathrm{E})$ mutation and expression. Brain Pathol 2013; 23: 574-83.

16. Schindler G, Capper D, Meyer J, et al. Analysis of BRAF V600E mutation in 1,320 nervous system tumors reveals high mutation frequencies in pleomorphic xanthoastrocytoma, ganglioglioma and extra-cerebellar pilocytic astrocytoma. Acta Neuropathol 2011; 121: 397-405.

17. Lanzafame S, Torrisi A, Barbagallo G, Emmanuele C, Alberio N, Albanese V. Correlation between histological grade, MIB-1, p53, and recurrence in 69 completely resected primary intracranial meningiomas with a 6 year mean follow-up. Pathol Res Pract 2000; 196: 483-8

18. Ozen O, Demirhan B, Altinors N. Correlation between histological grade and MIB-1 and p53 immunoreactivity in meningiomas. Clin Neuropathol 2005; 24: 219-24.

19. Oya S, Kawai K, Nakatomi H, Saito N. Significance of Simpson grading system in modern meningioma surgery: integration of the grade with MIB-1 labeling index as a key to predict the recurrence of WHO Grade I meningiomas. J Neurosurg 2012; 117: 121-8.

20. Goutagny S, Nault JC, Mallet M, Henin D, Rossi JZ, Kalamarides M. High incidence of activating TERT promoter mutations in meningiomas undergoing malignant progression. Brain Pathol 2014; 24: $184-9$.

21. Spiegl-Kreinecker S, Lotsch D, Neumayer K, et al. TERT promoter mutations are associated with poor prognosis and cell immortalization in meningioma. Neuro Oncol 2018; 20: 1584-93.

22. Sahm F, Schrimpf D, Olar A, et al. TERT promoter mutations and risk of recurrence in meningioma. J Natl Cancer Inst 2016; 108: djv377.

23. Ellison DW, Dalton J, Kocak M, et al. Medulloblastoma: clinicopathological correlates of SHH, WNT, and non-SHH/WNT molecular subgroups. Acta Neuropathol 2011; 121: 381-96.

24. Ramaswamy V, Remke M, Bouffet E, et al. Risk stratification of childhood medulloblastoma in the molecular era: the current consensus. Acta Neuropathol 2016; 131: 821-31. 

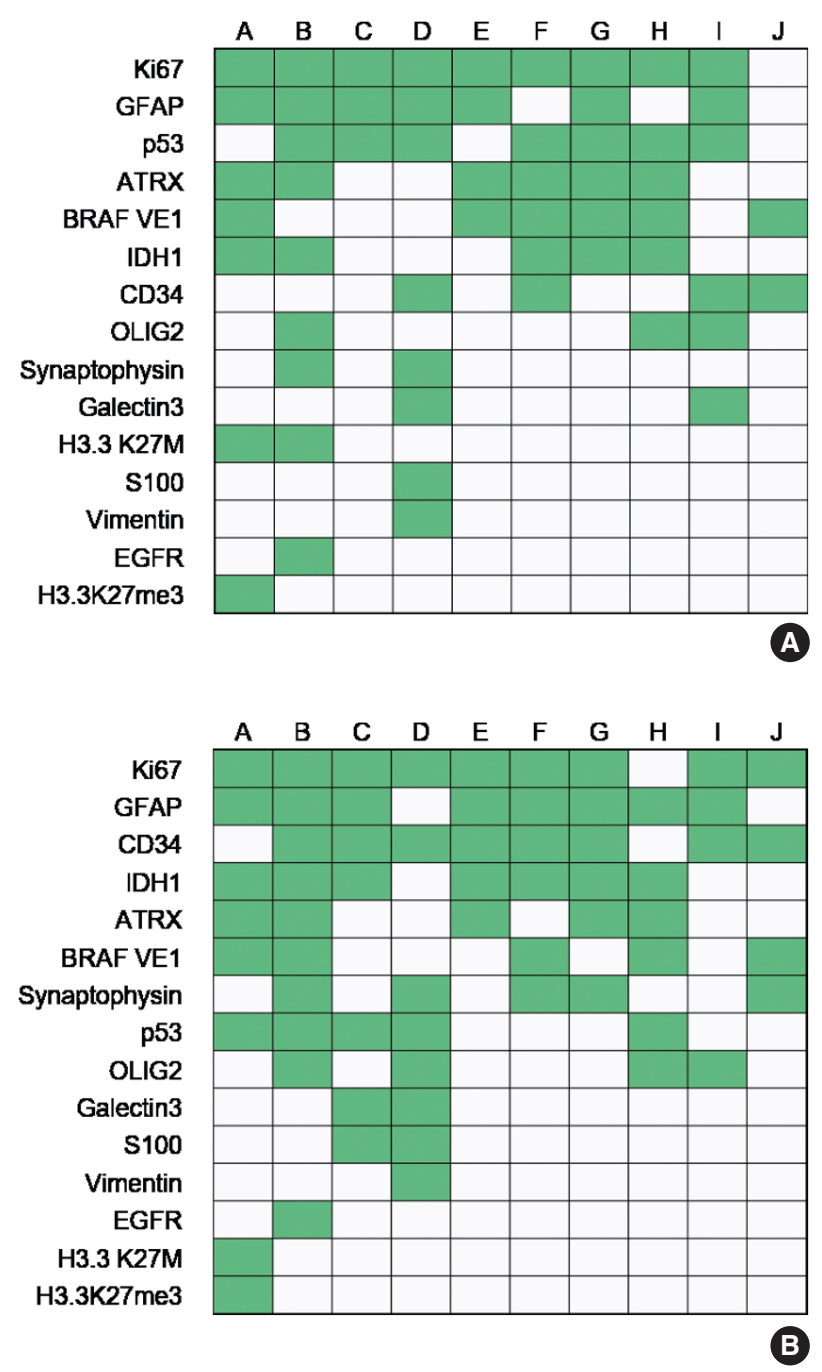

Supplementary Fig. S1. Immunohistochemistry markers used in pilocytic astrocytoma (A) and pleomorphic xanthoastrocytoma (B). Alphabet A-J represents respondents. GFAP, glial fibrillary acidic protein; ATRX, $\alpha$-thalassemia/mental retardation syndrome X-linked; IDH1, isocitrate dehydrogenase 1; OLIG2, oligodendrocyte transcription factor 2; EGFR, epidermal growth factor receptor. 

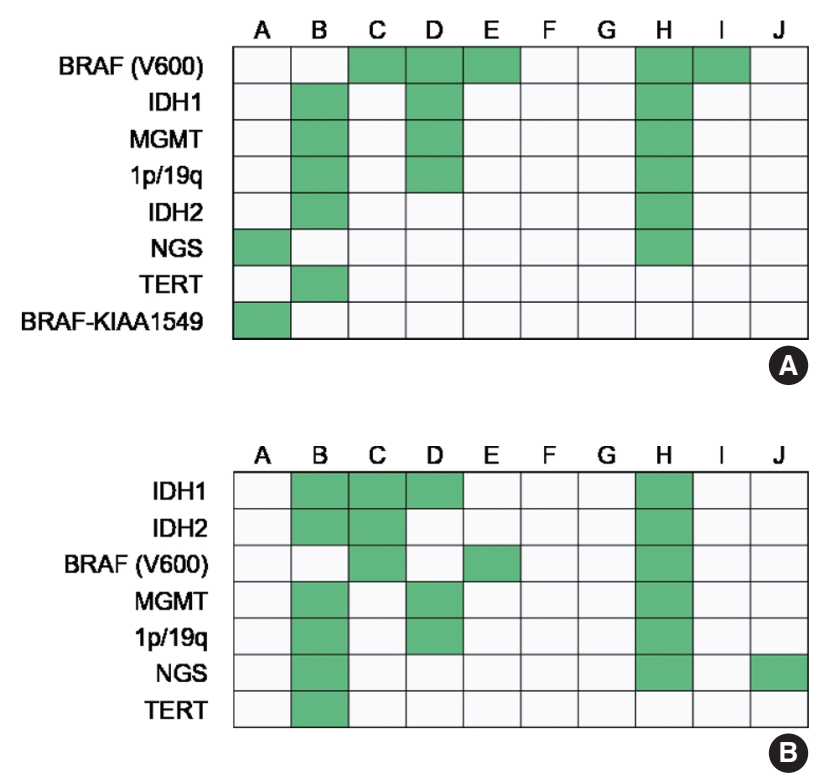

Supplementary Fig. S2. Molecular markers used in pilocytic astrocytoma (A) and pleomorphic xanthoastrocytoma (B). Alphabet A-J represents respondents. IDH1, isocitrate dehydrogenase 1; MGMT, $\mathrm{O}^{6}$-methylguanine-DNA methyltransferase; IDH2, isocitrate dehydrogenase 2; NGS, next-generation sequencing; TERT, telomerase reverse transcriptase. 